\title{
GEOTAIL observation of upstream ULF waves associated with lunar wake
}

\author{
Tomoko Nakagawa $^{1}$, Yoshinori Takahashi ${ }^{1 *}$, and Masahide Iizima ${ }^{2}$ \\ ${ }^{1}$ Tohoku Institute of Technology, 35-1 Yagiyama Kasumi-cho, Taihaku-ku, Sendai, Miyagi 982-8577, Japan \\ ${ }^{2}$ Tohoku University, Aramaki Aoba, Aoba-ku, Sendai Miyagi 980-8578, Japan
}

(Received April 1, 2003; Revised September 26, 2003; Accepted October 6, 2003)

\begin{abstract}
Left-handed, circular polarized ULF waves with frequency of $0.3-1.1 \mathrm{~Hz}$ were detected by GEOTAIL at 27 lunar radii upstream of the moon when the spacecraft was magnetically connected with the lunar wake. The wave was detected twice at 16:45-17:00 and 18:55-19:02 on October 25, 1994, when the spacecraft and the moon were on the dawn side of the Earth's magnetosphere. The ULF wave was propagating in a direction nearly parallel to the background magnetic field. The observed frequency and polarization are explained by reversal of polarization of right-handed, sunward-propagating electron whistler waves with frequencies above $1.4 \mathrm{~Hz}$ in the solar wind frame of reference, which were excited through the interaction with electron beams flowing in anti-sunward direction downstream of the lunar wake. The downstream flow of electron beam is explained by filtering effect of the potential drop at the boundary of the lunar wake. Low-energy components of electrons are reflected back by the potential drop, and the rest components, with energies higher than that of the electric potential penetrate through the wake. The velocity distribution of downstream electrons would be modified to have some bump or shoulder in energy range to form a beam, which is likely to excite whistler mode wave through cyclotron resonance. The lowest energy of the resonant electrons was calculated to be $0.96-2.5(\mathrm{keV})$ from the lower boundary of the detected frequency. The variation in the lowest frequency suggests that there are some regions of the lunar wake where potential drop is reduced.
\end{abstract}

Key words: Lunar wake, ULF wave in the solar wind, whistler mode wave, wake potential structure, cyclotron resonance.

\section{Introduction}

The lunar wake is a plasma cavity in the solar wind left on the anti-solar side of the moon as the solar wind particles were absorbed by the body of the moon (Schubert and Lichtenstein, 1974). The magnetic and plasma fluctuations characteristic of the lunar wake were extensively examined by Explorer 35 (Ness et al., 1968). The magnitude of the magnetic field was found to increase in the umbra, and an alternating pattern of increase and decrease was observed in the penumbra region. In addition to the magnetic structure, rapid fluctuation in magnetic field was observed in the vicinity of the wake when Explorer was magnetically connected to the lunar wake (Ness and Shatten, 1969).

The knowledge on the lunar wake has been refined by WIND spacecraft with its high-time resolution magnetic field and plasma experiments. WIND traversed the lunar wake at $\sim 6.8$ lunar radii downstream of the moon and evidenced that the wake extended to that distance (Owen et al., 1996). The magnitude of the magnetic field was enhanced in the shadow region and depressed just outside of the shadow. The plasma density was reduced from ambient solar wind value down to $0.5\left(\mathrm{~cm}^{-3}\right)$ (Ogilvie et al., 1996, Bosqued et al., 1996). Ogilvie et al. (1996) also showed the presence of field-aligned, cold ion beams refilling the lunar cavity, which

*Present address: Fusione Techno Solutions Co., Ltd., 1-24-1 Nishishinjuku, Shinjuku-ku, Tokyo, Japan.

Copy right(c) The Society of Geomagnetism and Earth, Planetary and Space Sciences (SGEPSS); The Seismological Society of Japan; The Volcanological Society of Japan; The Geodetic Society of Japan; The Japanese Society for Planetary Sciences. were accelerated by an electric field of the order of $2 \times 10^{-4}$ $(\mathrm{V} / \mathrm{m})$.

Prior to the entry into the lunar wake, the WIND spacecraft detected precursor ULF waves and energetic electron flows (Farrell et al., 1996). The ULF activity occurred upstream of the wake on field lines connected to the wake penumbra. The observed frequency $\omega_{O B S}$ varied stepwise depending on the abrupt change in the solar wind velocity $\mathbf{V}_{S W}$. Farrell et al. (1996) used a couple of $\omega_{O B S}$ corresponding to two different values of $\mathbf{V}_{S W}$ to obtain the angular frequency $\omega \simeq-12\left(\mathrm{rad} \mathrm{s}^{-1}\right)$ and the wave number $k \simeq 6 \times 10^{-5}\left(\mathrm{~m}^{-1}\right)$ in the solar wind frame, and concluded that the wave was a whistler mode wave. They estimated the energy of electrons that might be resonant with the whistler mode waves. The energy of the resonant electrons was calculated to be $500-1500(\mathrm{eV})$, while simultaneous observation of electrons showed the presence of counterstreaming component at $442(\mathrm{eV})$. Thus they suggested that electron beams reflected at the lunar wake were responsible for the generation of the whistler mode waves. Futaana et al. (2001) showed an enhancement of such backstream electrons in a velocity range below $13000\left(\mathrm{~km} \mathrm{~s}^{-1}\right)$ which suggests an acceleration of electrons by a potential drop of $475(\mathrm{~V})$.

In this paper, we report upstream ULF waves of $0.5-1.2$ $\mathrm{Hz}$ detected by GEOTAIL at 27 lunar radii upstream of the moon on October 25, 1994, when the spacecraft and the moon were both on the dawn side of the Earth's magnetosphere as shown in Fig. 1. The waves were observed twice at 16:45-17:00 and 18:38-19:02, when the interplanetary magnetic field line connected the moon and the spacecraft. 


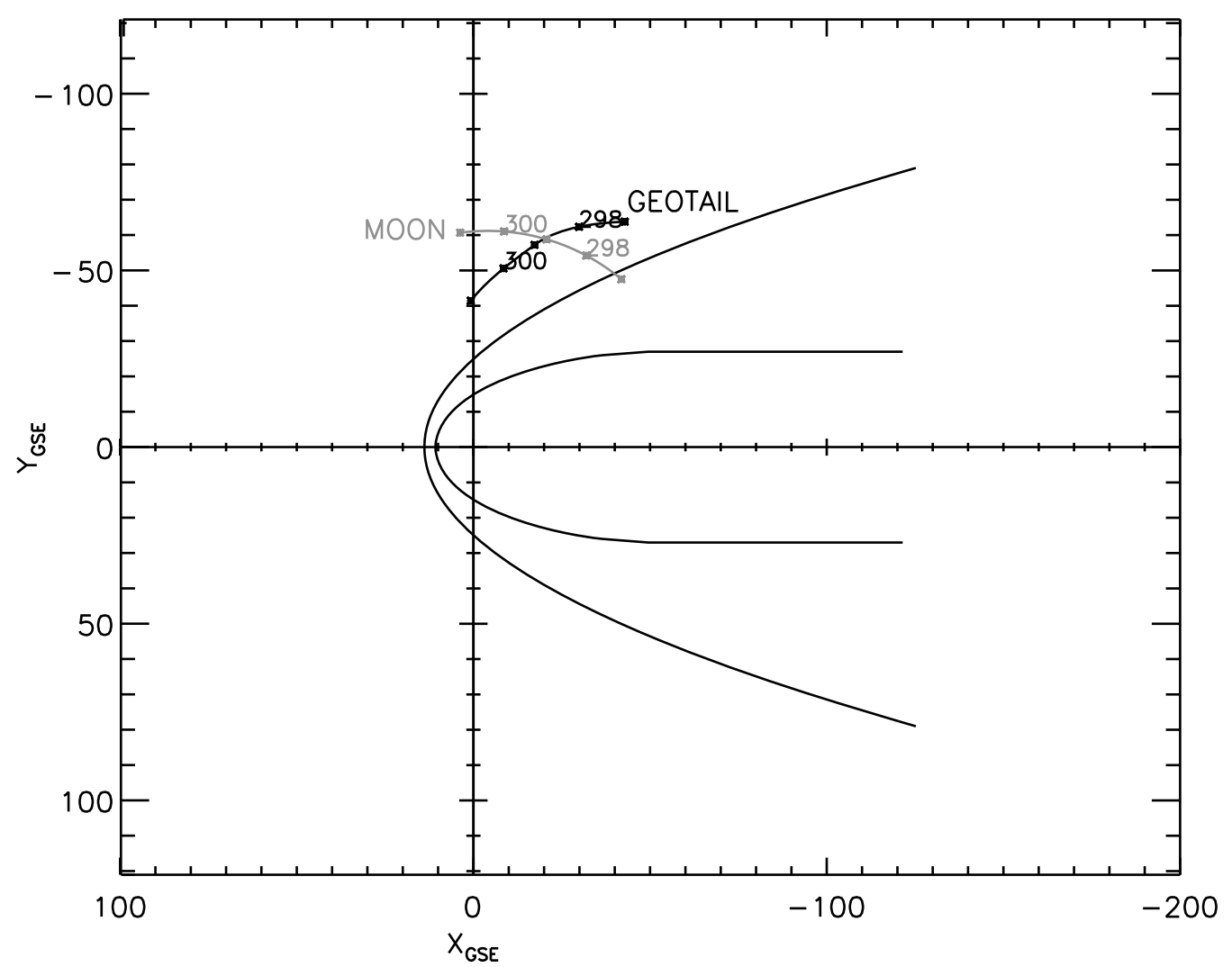

Fig. 1. The positions of the moon and the GEOTAIL in geocentric solar ecliptic coordinates during the period from October 24 (DOY 298 ), 1994 to October 26 (DOY 300), 1994, with projections of model bow shock and magnetopause surface. Both the moon and GEOTAIL were in the dawnside of the Earth. The original figure was adapted from ISTP Spacecraft Orbit Plots (http://www-istp.gsfc.nasa.gov/orbits/).

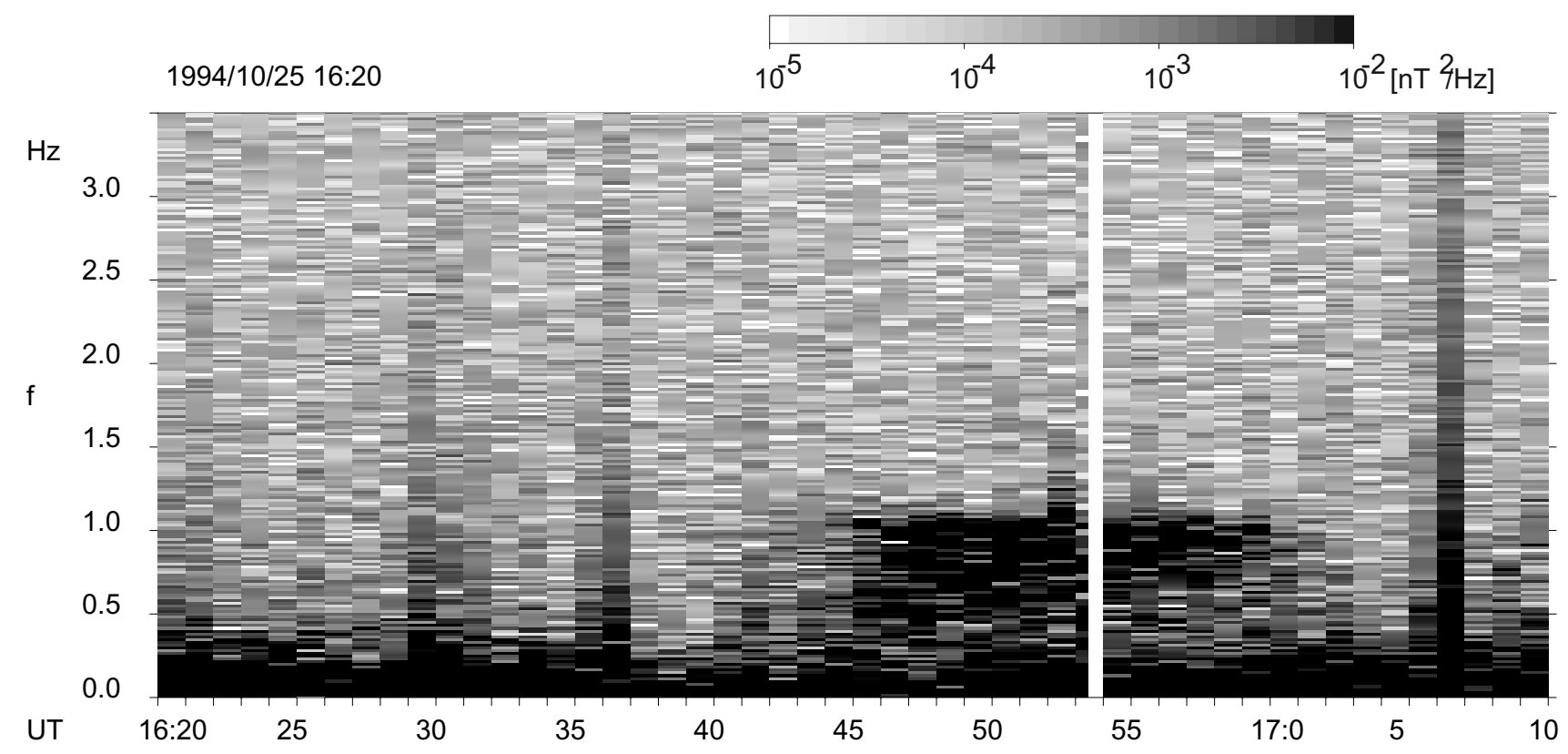

Fig. 2. The dynamic spectrum of the IMF $B_{z}$ component during the period from 16:20 to 17:10 on October 25 , 1994 . The wave activity associated with lunar wake was observed between 0.3 to $1.1 \mathrm{~Hz}$ during the period from 16:45 to 17:00. Each spectrum was calculated for each 1 minute period, except for the 26 second period 16:53:00-16:53:26 which was shortened by the data gap 16:53:26-16:53:41.

\section{Observation}

The magnetic field data used in this analysis were obtained by GEOTAIL/MGF instrument (Kokubun et al., 1994) at a sampling rate of 16 vectors/second. The 3 components and the magnitude of the magnetic field were Fourier trans- formed every 1 minute. Figure 2 shows a dynamic spectrum of the IMF $B_{z}$ component in the GSE coordinate system observed on October 25, 1994. During the period from 16:45 to $17: 00$, an enhancement of wave activity was found in a frequency range from 0.3 to $1.1 \mathrm{~Hz}$, in addition to the low 

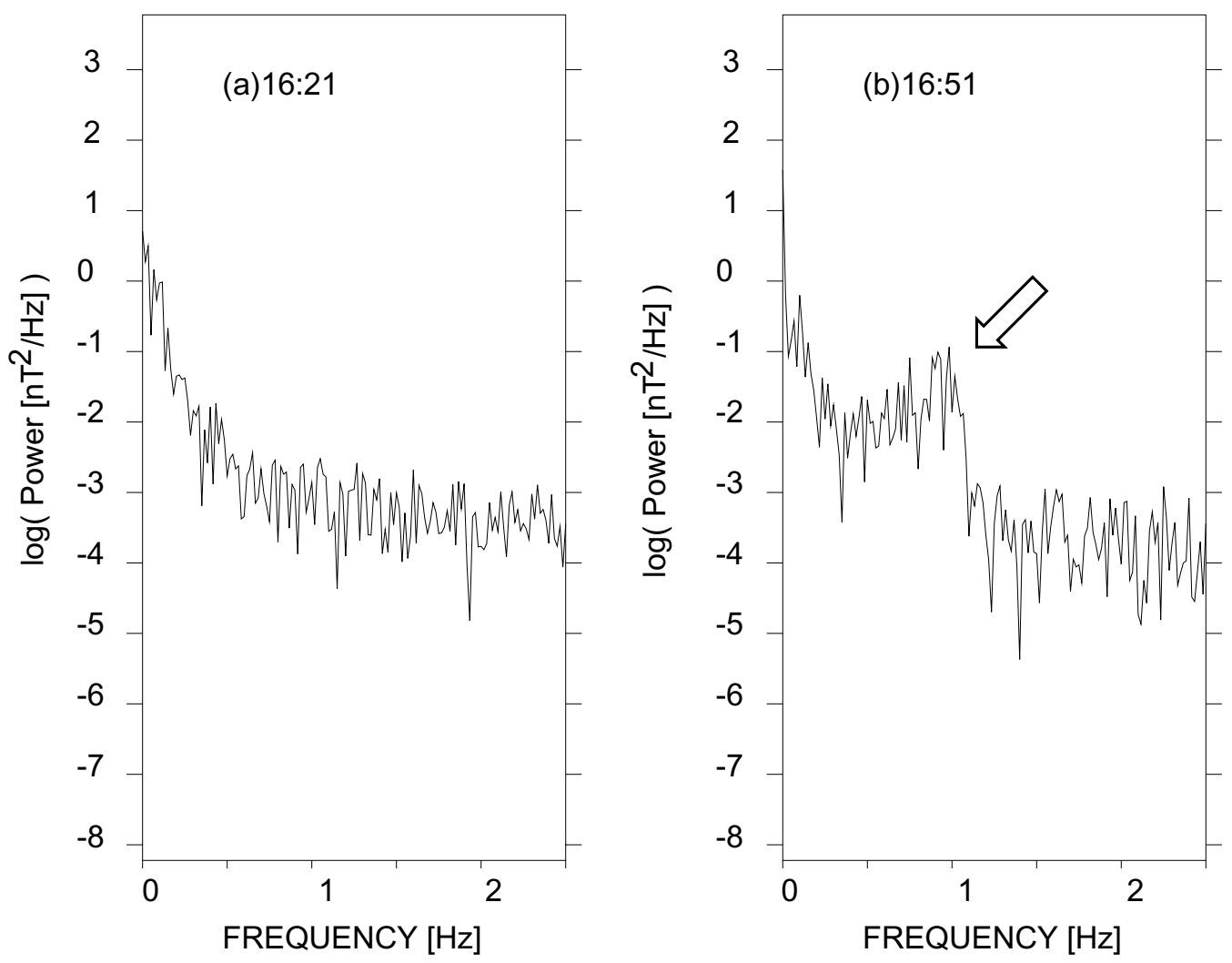

Fig. 3. The spectra of the magnetic fluctuations in (a) unperturbed solar wind obtained from 16:21 to 16:22, October 25, 1994, and (b) the ULF wave associated with the lunar wake, obtained from 16:51 to 16:52, October 25, 1994.

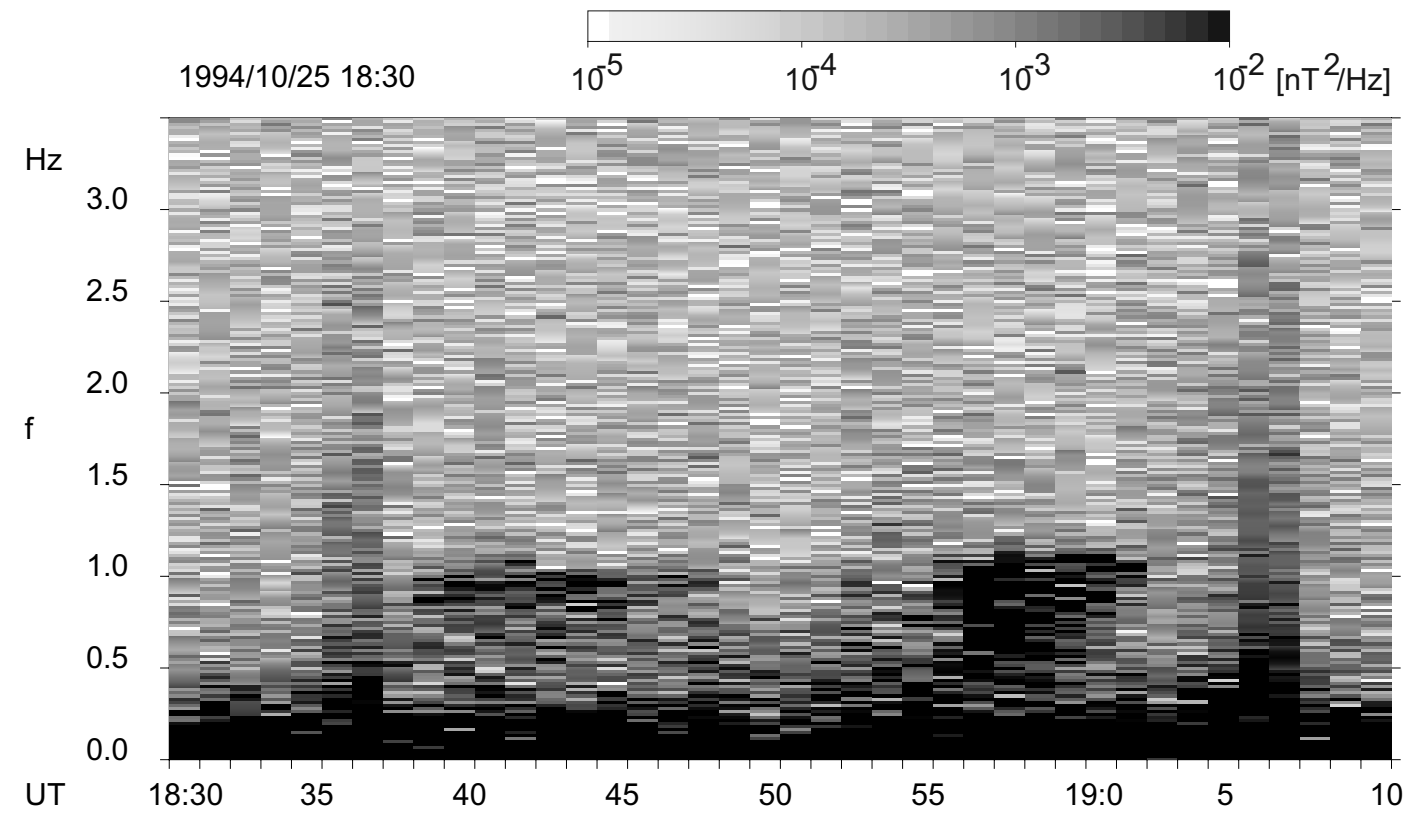

Fig. 4. The second example of the ULF wave encountered by GEOTAIL during the period from 18:55 to 19:02 on October 25, 1994. There was a wave activity between 0.3 and $1.1 \mathrm{~Hz}$ with a clear cut off at the upper boundary. Similar but less clear fluctuation was observed during the period from $18: 38$ to $18: 48$.

frequency $(f<0.3 \mathrm{~Hz})$ wave activities which are often observed in the solar wind.

Figure 3 compares the spectra of (a) the magnetic fluctuation in unperturbed solar wind, and (b) the ULF wave activity associated with the lunar wake. The frequency range of the wave activity in Fig. 3(b) shows a clear upper cutoff at 1.1
Hz. The lower boundary seem to be at around $0.3 \mathrm{~Hz}$, but it is less clear.

Figure 4 shows another example of the ULF wave encountered by GEOTAIL during the period from 18:55 to 19:02 on October 25, 1994; there was a wave activity in a frequency range $0.3-1.1 \mathrm{~Hz}$, again with a sharp upper cutoff at $1.1 \mathrm{~Hz}$. 

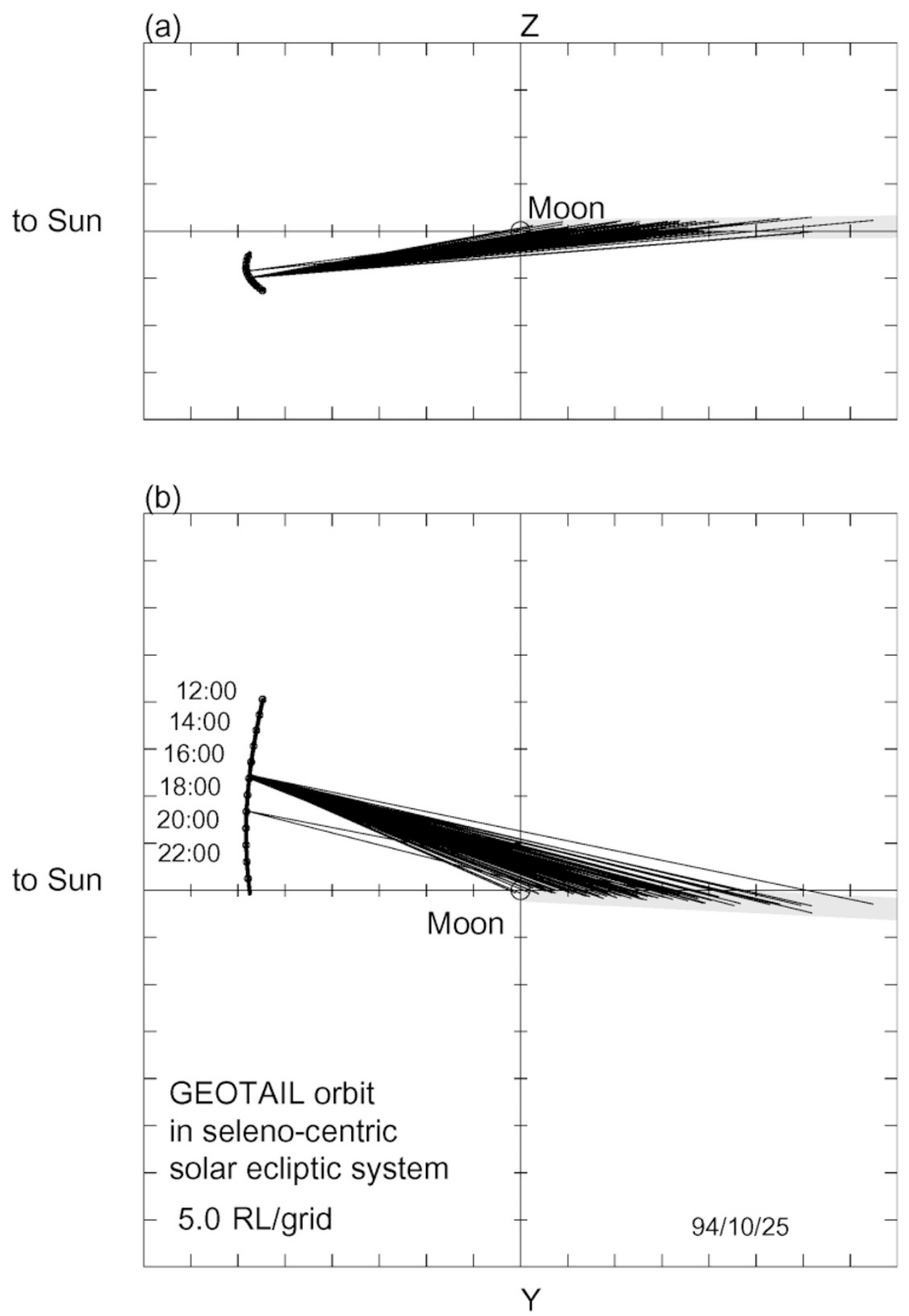

Fig. 5. Magnetic connection between the lunar wake and GEOTAIL. The 3-second magnetic field vectors only for the periods from 16:45 to 17:00 or from 18:55 to 17:02, are represented by straight lines if they crossed the modeled wake within $40 \mathrm{R}_{L}$ of the moon. The shaded area indicates the model lunar wake represented by a cylinder whose radius is the same as that of the moon, with an axis extends in the direction of the real-time solar wind behind the moon. (a) A meridional view. (b) An ecliptic view with the Sun on the left hand side.

The lower boundary which was higher than $0.7 \mathrm{~Hz}$ at the beginning of the event decreased gradually to be as low as 0.3 $\mathrm{Hz}$ at 18:57 in the middle of the duration of the wave activity, then it turned to increase to become higher than $1 \mathrm{~Hz}$ at the end of the event. The upper boundary of the frequency range remained the same. Similar feature was observed in the previous case given in Fig. 2.

Preceding the event, there is another wave activity at around $1 \mathrm{~Hz}$ during the period from 18:38 to 18:48. It also had upper cutoff at $1.1 \mathrm{~Hz}$ and similar signature in spectrum, but less clear.

The ULF waves were observed at 27 lunar radii upstream of the moon in the solar wind. The spacecraft did not enter the lunar wake itself, but was magnetically connected to the wake. Figure 5 shows the position of GEOTAIL with respect to the moon, the model lunar wake (the shaded area), and the magnetic field lines which connected the wake and the spacecraft. The position and the magnetic field are given in 


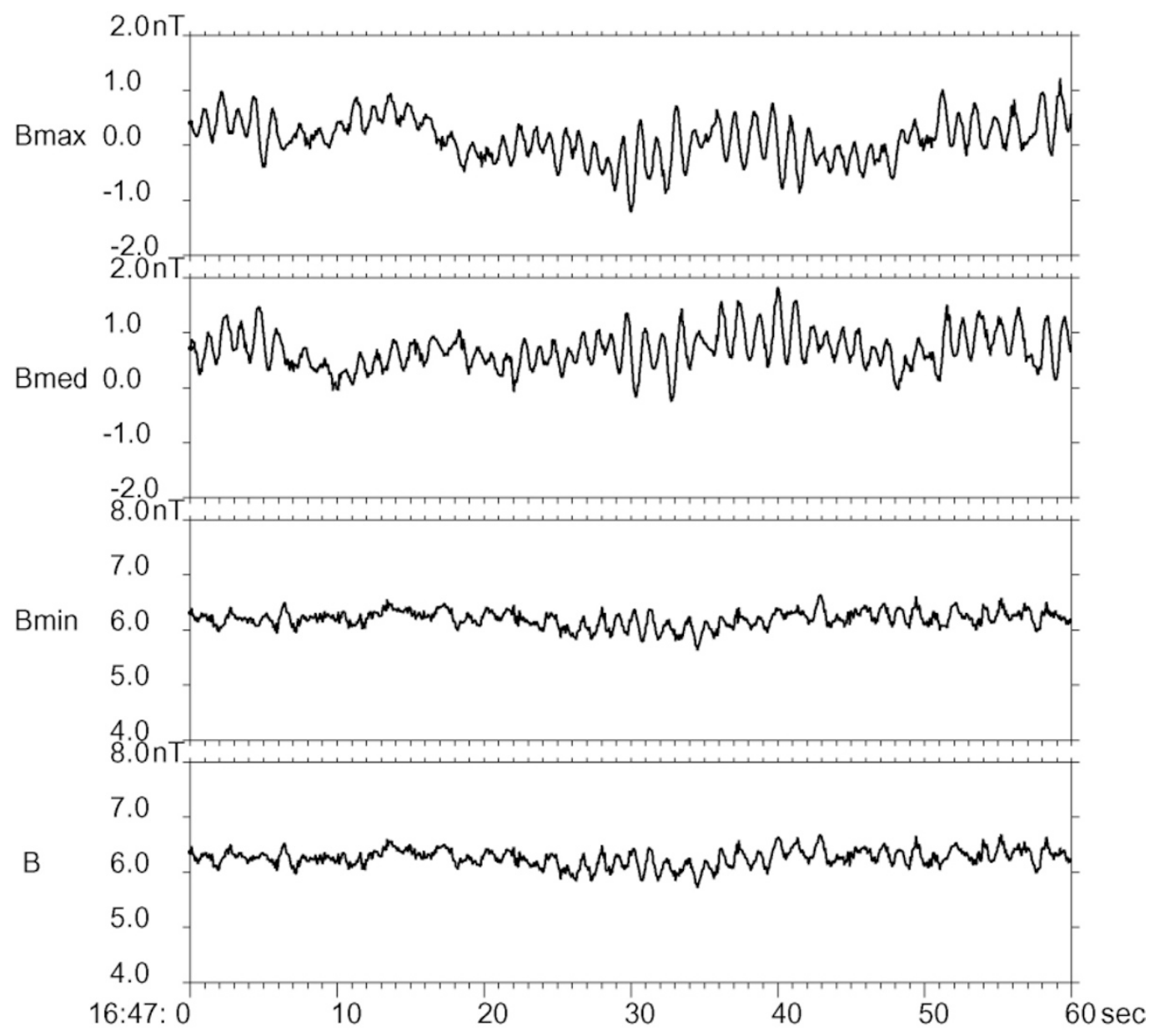

Fig. 6. An example of waveforms of the ULF waves in minimum variance coordinates. From top to bottom, the components along the direction of maximum, intermediate and minimum variance, and the magnitude of the magnetic field. No filter is applied after the magnetic field vector was sampled at $16 \mathrm{~Hz}$.

selenocentric solar ecliptic coordinates, and the Sun is on the left hand side of Fig. 5. The magnetic field was stable and the average was $(-5.8,1.8,0.7)(\mathrm{nT})$ for the period 16:45-17:00 and $(-6.0,2.6,0.4)(\mathrm{nT})$ for 18:55-19:00. The lunar wake is modeled with a cylinder whose radius is the same as that of the moon, with an axis extending downstream by 40 lunar radii from the center of the moon in the direction of the solar wind. The magnetic field lines are linearly extrapolated to see if they intersect the model lunar wake. It should be noted that the extrapolated field lines also intersected the Earth's bow shock throughout the half-day period 12:00-24:00 of October 25, 1994 displayed.

Figure 5 tells us that the magnetic field lines connected the spacecraft with the lunar wake. The magnetic connection allowed the upstream propagation of the ULF waves associated with the lunar wake. On the other hand, the magnetic connection is not the only condition for detection of the ULF wave. The magnetic field lines often connected the spacecraft with the lunar wake during the period from 12:00-24:00, but the ULF waves were detected in short, limited periods of 15,10 or 5 minutes described above. ULF waves were often absent even when GEOTAIL was magnetically connected to the wake. The intermittent detection of the wave activity suggests that some other condition is required for the wave to be accessible from GEOTAIL.
Figure 6 shows an example of waveforms of the $16 \mathrm{~Hz}$ magnetic field data for 1 minute period of the ULF wave. No bandpass filter was applied after the sampling. Note the enhancement of fluctuation with a frequency around $1 \mathrm{~Hz}$. The amplitude of the fluctuations in transverse components $B_{\max }$ and $B_{m e d}$ in the direction of maximum- and intermediatevariance were larger than that of the magnitude $B$ of the magnetic field, suggesting that the waves were propagating nearly parallel to the magnetic field.

The direction of the wave normal of the $1-\mathrm{Hz}$ ULF wave was calculated by minimum variance analysis (Sonnerup and Cahill, 1967) applied every 1 minute. As the value of midto minimum eigenvalue ratio $\left(\lambda_{\text {med }} / \lambda_{\text {min }}\right)$ was larger than 2.5 , we are confident that the direction was determined with sufficient accuracy (Lepping and Behannon, 1980). Figure 7 shows the angle between the background magnetic field and the wave number vector. During the period from 16:45 to 17:00, the angle was on average as small as 10 degrees, indicating that the wave was propagating nearly parallel to the magnetic field. The situation was the same for the other cases; the angle between the wave number vector and the background magnetic field was less than 14 degrees during the periods from 18:55 to 19:01, and from 18:38 to $18: 46$.

The magnetic fluctuation of the ULF wave was found to be left-hand polarized with respect to the background mag- 


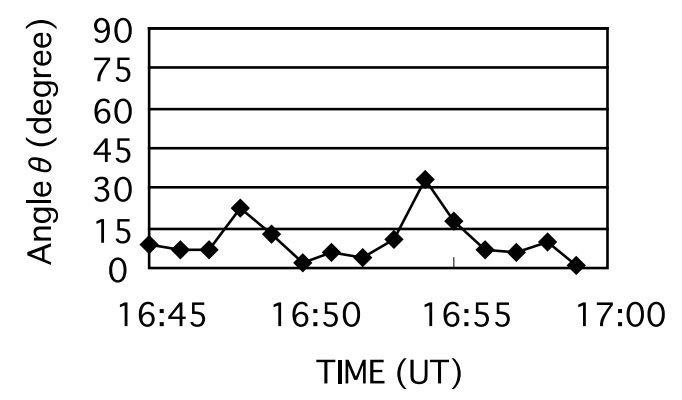

Fig. 7. The angle between the background magnetic field and the wave number vector of the $1-\mathrm{Hz}$ ULF wave calculated by minimum variance analysis applied every 1 minute. The angle remained as small as $10 \mathrm{de}-$ grees, although there were occasional excursions to 33 degrees at maximum.

netic field in the spacecraft frame of reference, i.e., rotating in the opposite direction of the gyration of electrons. Figure 8 shows an example of the hodogram of the magnetic field in minimum variance coordinates. Left-handed, circular polarization is clearly seen. Throughout the periods of 16:45-17:00 and 18:55-19:02, the ULF waves showed lefthand polarization.

\section{Resonance Condition between the ULF Wave and Electrons}

\subsection{Summary of observations}

The properties of the ULF waves detected by GEOTAIL upstream of the lunar wake are summarized as follows:

i) The frequency ranged from 0.3 to $1.1 \mathrm{~Hz}$ with sharp upper boundary.

ii) The ULF wave propagated in a direction nearly parallel to the background magnetic field. iii) The ULF wave was left-hand polarized as measured in the spacecraft frame of reference.

iv) The ULF waves were detected when GEOTAIL was magnetically connected to the lunar wake, although the wave activity often disappeared in the same configuration of the magnetic field.

v) The lower boundary of the frequency of the ULF waves was variable and it was lowest in the middle of each event.

\subsection{Polarity reversal of sunward-propagating waves}

The ULF wave was detected by GEOTAIL upstream from the moon only when the magnetic field connected the spacecraft and the lunar wake. It suggests that the wave was propagating away from the wake along the magnetic field line toward the Sun against the solar wind flow. As Fairfield (1974) suggested concerning the upstream wave from the bow shock, the most probable candidate for such ULF wave is the whistler mode, since it has a group velocity greater than the solar wind velocity. The whistler mode wave is right-hand polarized in the frame of the solar wind plasma, however, waves observed by a spacecraft undergo a Doppler shift to be detected at an angular frequency

$$
\omega_{O B S}=\omega+\mathbf{k} \mathbf{V}_{S W}
$$

by a spacecraft in a rest frame, where $\omega$ and $\mathbf{k}$ are the angular frequency and wave number vector in the solar wind frame of reference, respectively, and $\mathbf{V}_{S W}$ is the solar wind velocity with respect to the spacecraft. The observed frequency $\omega_{O B S}$ turns to be negative when $\mathbf{k}$ is in the upstream direction and $\left|\mathbf{k} \mathbf{V}_{S W}\right|$ is larger than $\omega$, indicating that the observed polarity is reversed.

For the case presently analyzed, the solar wind velocity $\mathbf{V}_{S W}$ was $(-501,25,6)\left(\mathrm{km} \mathrm{s}^{-1}\right)$ and the direction of $\mathbf{k}$ was $\frac{\mathbf{k}}{|\mathbf{k}|} \sim(0.92,-0.35,-0.13)$ for $16: 50-16: 51$, thus it
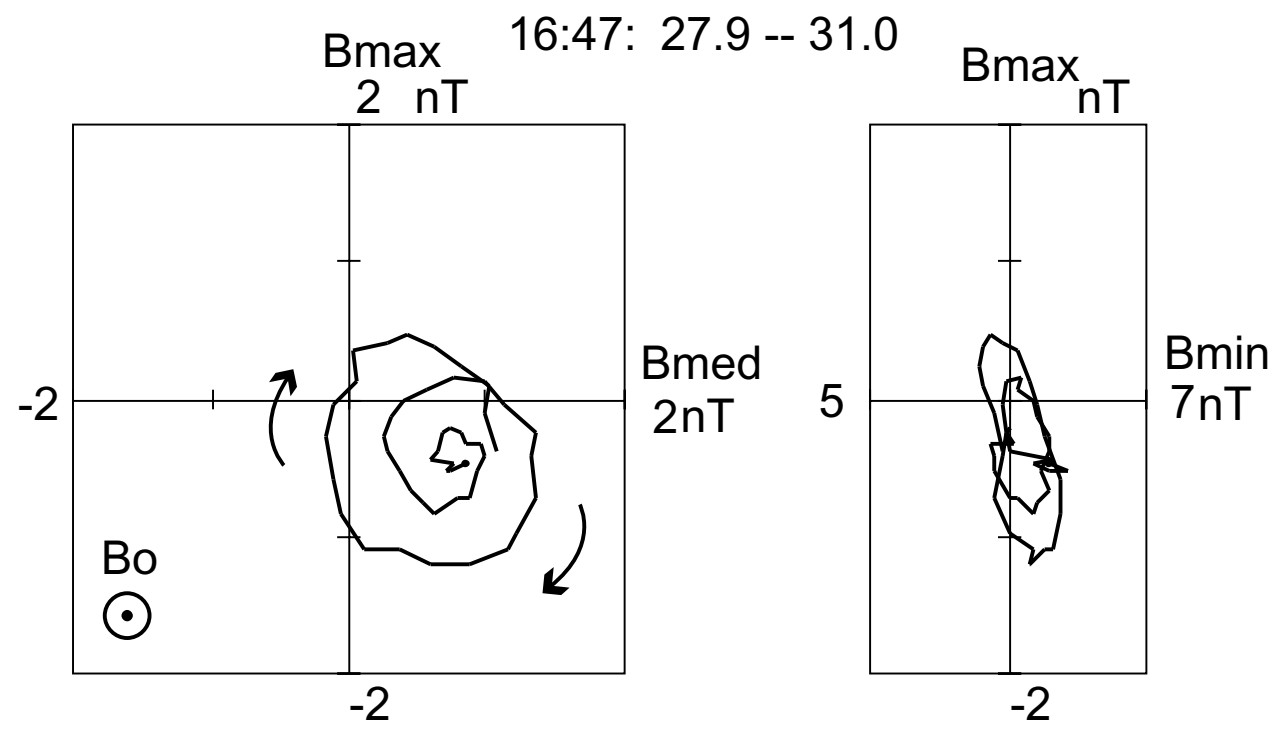

Fig. 8. A hodogram of the magnetic field during a $3 \mathrm{sec}$ period from 16:47:27.9 to $16: 47: 31.0$ sampled at $16 \mathrm{~Hz}$, displayed in minimum variance coordinates determined for 1-minute period from 16:47:00 to 16:48:00. The direction of the background magnetic field was approximately from back to front side of the paper, $\mathbf{B}_{0} \sim(6.2,0.7,0.1)(\mathrm{nT})$ in minimum variance coordinates. Left-hand polarization was detected throughout the period of enhanced ULF wave. 
Table 1. Polarization detected at GEOTAIL for combinations of polarization and orientation of $\mathbf{k}$ vector in solar wind frame.

\begin{tabular}{lllll}
\hline \multicolumn{2}{l}{ In solar wind frame } & \multicolumn{2}{l}{ In GEOTAIL frame } & Remark \\
Polarization & $\mathbf{k}$ & $\omega_{O B S}$ & Polarization to be detected & \\
\hline $\begin{array}{l}\text { right-hand } \\
\text { (electrons) }\end{array}$ & sunward & $\omega-\left|\mathbf{k} \mathbf{V}_{S W}\right|$ & $\begin{array}{l}\text { left-handed if } \omega<\left|\mathbf{k} \mathbf{V}_{S W}\right| \\
\text { right-handed if } \omega>\left|\mathbf{k} \mathbf{V}_{S W}\right|\end{array}$ & $\begin{array}{l}\text { OK } \\
\text { NG }\end{array}$ \\
\cline { 2 - 5 } & anti-sunward & $\omega+\left|\mathbf{k} \mathbf{V}_{S W}\right|$ & right-handed & NG \\
\hline $\begin{array}{l}\text { left-hand } \\
\text { (ions) }\end{array}$ & sunward & $\omega-\left|\mathbf{k} \mathbf{V}_{S W}\right|$ & $\begin{array}{l}\text { right-handed if } \omega<\left|\mathbf{k} \mathbf{V}_{S W}\right| \\
\text { left-handed if } \omega>\left|\mathbf{k} \mathbf{V}_{S W}\right|\end{array}$ & $\begin{array}{l}\text { NG } \\
\text { prohibited }\end{array}$ \\
\cline { 2 - 5 } & anti-sunward & $\omega+\left|\mathbf{k} \mathbf{V}_{S W}\right|$ & left-handed & inaccessible \\
\hline
\end{tabular}

Table 2. Cycrotron resonance with electrons

\begin{tabular}{llll}
\hline \multicolumn{2}{l}{ Wave in solar wind frame } & & \\
Polarization & $\mathbf{k}$ & Electron beam & Resonance condition \\
\hline right-hand & sunward & sunward & $\omega-\left|\mathbf{k V}_{B E A M}\right|-\left|\mathbf{k} \mathbf{V}_{S W}\right|=n \Omega_{e}$ \\
(electrons) & $\omega$ & $\left(\mathbf{k} \mathbf{V}_{B E A M}>0\right)$ & $\mathrm{n}<0$, opposite to electron cycrotron \\
& $\left|\mathbf{k} \mathbf{V}_{S W}\right|$ & & motion \\
\cline { 3 - 4 } & & anti-sunward & $\omega+\left|\mathbf{k} \mathbf{V}_{B E A M}\right|-\left|\mathbf{k} \mathbf{V}_{S W}\right|=n \Omega_{e}$ \\
& $\left(\mathbf{k} \mathbf{V}_{B E A M}<0\right)$ & $\mathrm{n}>0$ if \\
& & $\left|\mathbf{k} \mathbf{V}_{B E A M}\right|>\left|\mathbf{k} \mathbf{V}_{S W}\right|-\omega$ \\
\hline
\end{tabular}

$\Omega_{e}$ : electron cycrotron frequency

was possible that a right-hand wave in the solar wind frame was observed as a left-hand polarized wave in the GEOTAIL frame only if the phase speed was smaller than $470\left(\mathrm{~km} \mathrm{~s}^{-1}\right)$.

Table 1 lists possible combinations of polarization and orientation of wave number vector $\mathbf{k}$ of candidate waves in the solar wind frame, together with resulting frequency $\omega_{O B S}$ and polarization as seen from GEOTAIL. The detection of left-hand polarized wave is explained by

i) sunward-propagation of right-hand polarized wave whose phase speed was lower than the solar wind velocity component along the wave vector,

ii) sunward-propagation of left-hand polarized wave whose phase speed was higher than the solar wind velocity component along the wave vector, or

iii) anti-sunward propagation of left-hand polarized wave.

The second candidate is improbable because no left-hand wave is allowed to exist in that frequency range. According to Eq. (1), the frequency in the solar wind frame $\omega=\omega_{O B S}-$ $\mathbf{k} \mathbf{V}_{S W}=\omega_{O B S}+\left|\mathbf{k} \mathbf{V}_{S W}\right|$ becomes larger than the observed frequency $\omega_{O B S}=2.5-7.5\left(\mathrm{rad} \mathrm{s}^{-1}\right)$, which is much higher than the ion cyclotron frequency $\Omega_{i}=0.6\left(\mathrm{rad} \mathrm{s}^{-1}\right)$ for the background field $|B| \sim 6.2$ (nT) for this case. In the plasma wave theory, no left-hand polarized wave is allowed to exist in the frequency range $\Omega_{e}>\omega>\Omega_{i}$.

The last candidate is inaccessible from GEOTAIL unless the wave is generated upstream of the spacecraft.

Thus the most probable explanation of the detection of left-handed wave is reversal of polarization of the sunwardpropagating waves which are right-hand polarized in the solar wind frame.

The reversal of the polarization does not occur unless the wave propagates against the solar wind. Thus the wake- related ULF waves with left-hand polarization must be observed only in upstream of the lunar wake.

\subsection{Resonance condition for electron beams}

In order that the sunward-propagating wave become resonant with gyrating electrons, the electric field in the electron frame of reference must be right-hand polarized. Table 2 summarizes the resonance condition between right-hand polarized waves and electrons that can account for the left-hand polarization in the GEOTAIL frame of reference.

As recognized in Table 2, the resonant electron beam must flow downstream in the solar wind stream. For such electrons, the resonant condition with sunward-propagating wave is

$$
\omega+\left|\mathbf{k} \mathbf{V}_{B E A M}\right|-\left|\mathbf{k} \mathbf{V}_{S W}\right|=n \Omega_{e} \quad(n= \pm 1, \pm 2, \pm 3, \ldots)
$$

where the harmonic number $n$ becomes positive if $\left|\mathbf{k} \mathbf{V}_{B E A M}\right|>\left|\mathbf{k} \mathbf{V}_{S W}\right|-\omega$. It is easily satisfied by electrons whose speed is larger than the difference between the solar wind speed and the phase speed of the whistler mode wave. Assuming $n=1$, the energy of the electron beam is obtained from

$$
\left|\mathbf{k} \mathbf{V}_{B E A M}\right|=\left|\mathbf{k} \mathbf{V}_{S W}\right|-\omega+\Omega_{e} .
$$

By substituting Eq. (1), we can rewrite Eq. (3) as

$$
\left|\mathbf{k} \mathbf{V}_{B E A M}\right|=-\omega_{O B S}+\Omega_{e}
$$

On the other hand, Farrell et al. (1996) suggested cycrotron resonance of whistler mode waves with reflected electrons, but the resonance condition for sunward flowing electrons with sunward-propagating wave in the solar wind frame of reference is

$$
\omega-\left|\mathbf{k} \mathbf{V}_{B E A M}\right|-\left|\mathbf{k} \mathbf{V}_{S W}\right|=\omega_{O B S}-\left|\mathbf{k} \mathbf{V}_{B E A M}\right|=n \Omega_{e}
$$




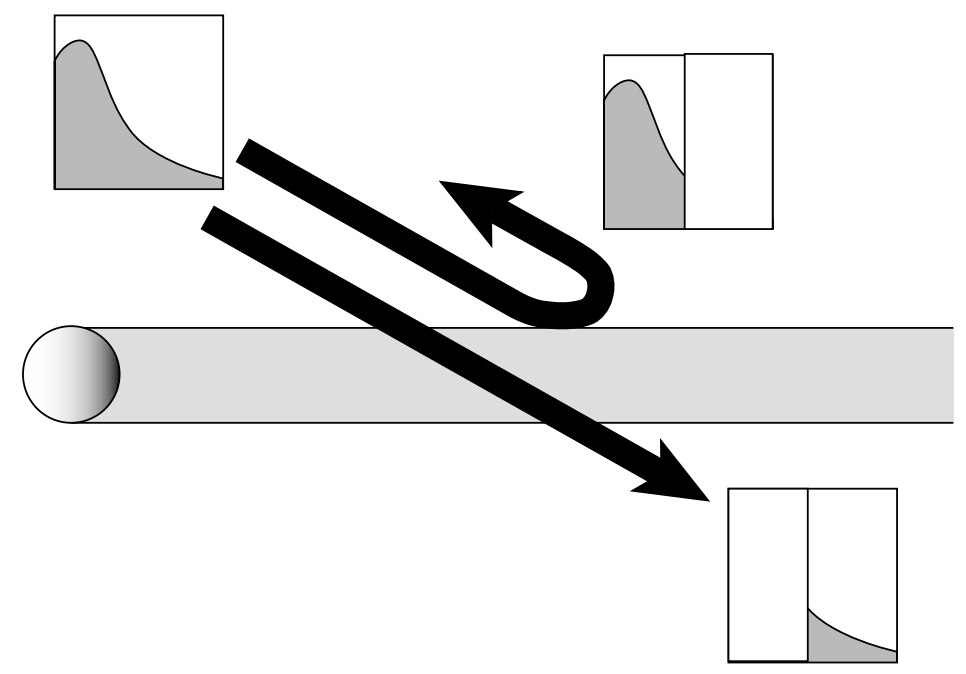

Fig. 9. A schematic illustration of velocity distribution function of electrons modified by the presence of the lunar wake. Low-energy components are reflected back by the potential difference at the boundary of the wake, whereas high-energy components that can overcome the potential difference pass through the lunar wake into the downstream solar wind, where they undergo resonant interaction with waves.

As evidenced by the polarity reversal, $\omega_{O B S}$ is negative, thus $n$ in Eq. (5) becomes negative, indicating that the wave is rotating in the opposite direction of cycrotron motion of electrons when observed from electrons moving sunward at a velocity $\mathbf{V}_{B E A M}$. That is, the reflected electrons outstrip the upstream-propagating wave and observe left-hand polarized field. Thus effective energy exchange is not expected between the sunward-propagating wave and reflected electrons propagating in sunward direction.

\subsection{Anti-sunward streaming electron beams}

The electron beam flowing downstream in the solar wind does not conflict with the idea of reflection of electrons at the boundary of the lunar wake. Figure 9 illustrates the idea. Low-energy components of electrons are reflected back by the potential drop at the lunar wake, and the rest components, with energies higher than that of the electric potential at the boundary of the lunar wake, penetrate through the wake into the downstream solar wind, where they are coupled with the sunward-propagating wave. The lunar wake behaves like a filter that percolates only high-energy electrons. The velocity distribution of downstream electrons would be modified to have some bump or shoulder, which is likely to cause some beam driven instability.

\subsection{Resonance with whistler mode waves}

The energy of resonant electrons as well as the frequency and wave number of the resonant wave are obtained from Eqs. (1) and (3) combined with the dispersion relation for the whistler mode wave

$$
\frac{c k}{\omega}=\frac{\omega_{p e}}{\sqrt{\omega\left(\Omega_{e} \cos \theta-\omega\right)}},
$$

where $c$ is the speed of light, $\omega_{p e} \equiv \sqrt{\frac{n_{e} q^{2}}{m_{e} \epsilon}}=2 \pi \times$ $18(\mathrm{kHz})$ is the plasma frequency deduced from the frequency of Langmuir wave detected by GEOTAIL/PWI instrument (Matsumoto et al., 1994, http: //www.kurasc.kyoto-u.ac.jp/gtlpwi), $\Omega_{e} \equiv \frac{q B_{0}}{m_{e}}=2 \pi \times 174(\mathrm{~Hz})$ is the electron cyclotron frequency calculated from the mass $m_{e}$ of electrons, the unit charge $q$, and the magnitude of the background magnetic field $B_{0}=6.2(\mathrm{nT})$. The angle $\theta$ between the wave number vector $\mathbf{k}$ and the background magnetic field $\mathbf{B}_{0}$ was small for the present cases.

Figure 10 illustrates the dispersion curve (6) for parallel propagation $\theta=0$ and the condition (3) for the electron cycrotron resonance. In the $\omega$-k diagram, Equation (3) appears as a straight line

$$
\omega=\Omega_{e}-\left(V_{B E A M} \cos \theta_{k e}-V_{S W} \cos \theta_{k S}\right) k
$$

with a negative inclination $-\left(V_{B E A M} \cos \theta_{k e}-V_{S W} \cos \theta_{k S}\right)$, where $\theta_{k e}$ is the angle between the wave number vector $\mathbf{k}$ and the beam velocity $\mathbf{V}_{B E A M}$, and $\theta_{k S} \simeq 20^{\circ}$ is the angle between $\mathbf{k}$ and the solar wind velocity $\mathbf{V}_{S W}$. As the wave number vector $\mathbf{k}$ was almost aligned with $\mathbf{B}_{0}$, the angle $\theta_{k e}$ is thought to be small under the assumption that the electron beam was nearly aligned with the background field $\mathbf{B}_{0}$. The inclination of the line of Eq. (7) is negative, because $V_{B E A M} \cos \theta_{k e}$ is supposed to be larger than $V_{S W} \cos \theta_{k S}$. Indeed, Farrell et al.(1996) estimated the energy of the resonant beam to be $500-1500(\mathrm{eV})$ which corresponds to 1.3 $\times 10^{7}-2.3 \times 10^{7}\left(\mathrm{~m} \mathrm{~s}^{-1}\right)$, while $V_{S W}$ was $5.0 \times 10^{5}\left(\mathrm{~m} \mathrm{~s}^{-1}\right)$. The inclination becomes steeper as the electron beam becomes faster.

The cyclotron resonance occurs at the intersection of the dispersion curve and the negatively-inclined straight line (7) in Fig. 10. Around the resonant point, the group velocity $V_{g}=\frac{\partial \omega}{\partial k}$ is larger than the phase velocity $V_{p h}=\frac{\omega}{k}$. The resonant wave is detectable from GEOTAIL when the group velocity is fast enough to propagate upstream against the solar wind flow

$$
V_{S W}<V_{g} \cos \theta_{k S}
$$

and the detected polarity becomes left-hand if $\omega<\left|\mathbf{k} \mathbf{V}_{S W}\right|$, that is,

$$
V_{p h}<V_{S W} \cos \theta_{k S}
$$

The inequality (8) gives the lower limit of $\omega$, while the inequality (9) gives the upper limit of $\omega$. In Fig. 10, the resonant wave must be on the dispersion curve between the two 


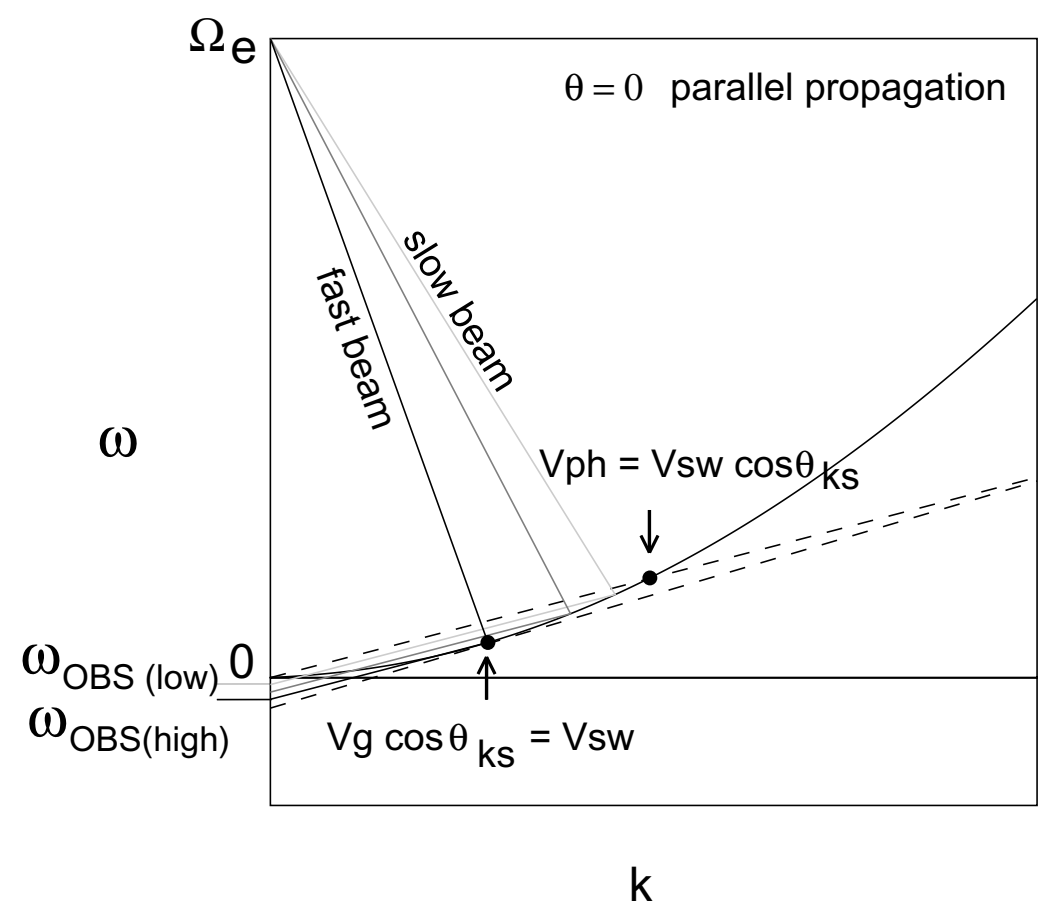

Fig. 10. A schematic illustration of the dispersion curve (6) and the condition (3) for the electron cyclotron resonance of $n=1$ which appears as straight lines with negative inclinations $-\left(V_{B E A M} \cos \theta_{k e}-V_{S W} \cos \theta_{k S}\right)$. The inclination becomes steeper as the electron beam becomes faster. The light line represents a slow beam. The resonance occurs at the intersection of the dispersion curve and the negatively-inclined line. The resonant wave then undergoes a Doppler shift according to Eq. (1), represented by the lines with positive inclinations $V_{S W} \cos \theta_{k S}$, and detected by a spacecraft at an angular frequency $\omega_{O B S}$. Because of the polarization reversal, large $\omega$ in the solar wind frame is detected as small $\left|\omega_{O B S}\right|$ in GEOTAIL frame of reference. Dashed lines are drawn to indicate the upper limit of $\omega$ where $V_{p h}=V_{S W} \cos \theta_{k S}$ and the lower limit where $V_{g} \cos \theta_{k S}=V_{S W}$.

dashed lines representing the boundaries of the inequalities (8) and (9).

By substituting the observed parameters $V_{S W}=502(\mathrm{~km}$ $\mathrm{s}^{-1}$ ) and $\cos \theta_{k S}=0.94$ into the inequalities (8) and (9), we can estimate the range of $\omega$ and $k$ of the resonant wave as

$$
0.82 \times 10^{-2} \Omega_{e}<\omega<2.7 \times 10^{-2} \Omega_{e}
$$

and

$$
9.4\left(\frac{\Omega_{e}}{c}\right)<k<17\left(\frac{\Omega_{e}}{c}\right)
$$

which correspond to frequency of $1.4-4.7 \mathrm{~Hz}$ and wavelength of 100-180 (km).

From the range of $\omega$ and $k$ thus obtained, we can estimate the inclination of the line of Eq. (7) in Fig. 10. The line of Eq. (7) connects a point $(k, \omega)$ on the dispersion curve in the range described above and the point $\left(0, \Omega_{e}\right)$, the inclination of the line is obtained as

$$
0.057 c<\left(V_{B E A M} \cos \theta_{k e}-V_{S W} \cos \theta_{k S}\right)<0.10 c,
$$

which results in the energy of the resonant electron beam $\frac{1}{2} m V_{B E A M}^{2}$ of the order of $0.82-2.5(\mathrm{keV})$. Much faster electrons out of this range may be in resonance with whistler mode wave, but such wave cannot be detected by GEOTAIL because its group velocity is too small to propagate against the solar wind stream.

\subsection{Upper boundary of the observed waves}

The resonant wave should be observed at an angular frequency $\omega_{O B S}$ determined by Eq. (1), which appears as a straight line with a positive inclination $V_{S W} \cos \theta_{k S}$ in
Fig. 10. Figure 11 shows a close-up of Fig. 10 for the parameters observed. Because of the polarity reversal, the upper limit of the observed frequency $\left|\omega_{O B S}\right|$ corresponds to the lower limit of $\omega$ determined by the condition (8) for the group velocity $V_{g}$. By substituting $\omega=0.82 \times 10^{-2} \Omega_{e}$ and $k=9.4\left(\frac{\Omega_{e}}{c}\right)$ into Eq. (1), the upper limit of $\left|\omega_{O B S}\right|$ is calculated to be $1.1 \mathrm{~Hz}$, which agrees with the observation.

The lower limit of $\omega$ depends only on the dispersion curve (6) and the condition (8), the upper boundary of the observed frequency $\left|\omega_{O B S}\right|$ remains the same as far as the solar wind condition represented by $\Omega_{e}, \omega_{p e}, \mathbf{V}_{S W}$ and the angle $\theta_{k S}$ remains unchanged. Actually the solar wind condition was almost constant as recognized in Fig. 12. This is the reason why the upper boundary of the observed frequency $\omega_{O B S}$ was constatnt while the lower boundary was variable.

\subsection{Lower boundary of the observed waves}

On the other hand, the lower limit of the observed frequency $\left|\omega_{O B S}\right|$ was higher than that expected from the inequality (9) as recognized in Fig. 11. The upper dashed line represents the inequality (9) which results in $\left|\omega_{O B S}\right| \sim 0$, while minimum value of the observed angular frequency $\omega_{O B S \text { (minimum) }}$ deviated significantly from 0 . Rather, it is considered that the lower limit of the observed frequency $\left|\omega_{O B S}\right|$ is controlled by the lower limit of the energy of the resonant electrons.

We can estimate the lowest energy of the resonant electrons by using the lowest frequency $\left|\omega_{O B S}\right|$. The light line in Fig. 11 represents Eq. (1) with lowest $\left|\omega_{O B S}\right|$ of $0.3 \mathrm{~Hz}$ detected in the middle of each event. The line intersects the dispersion curve at $(k, \omega)=\left(15.7 \frac{\Omega_{e}}{c}, 2.3 \times 10^{-2} \Omega_{e}\right)$, thus 


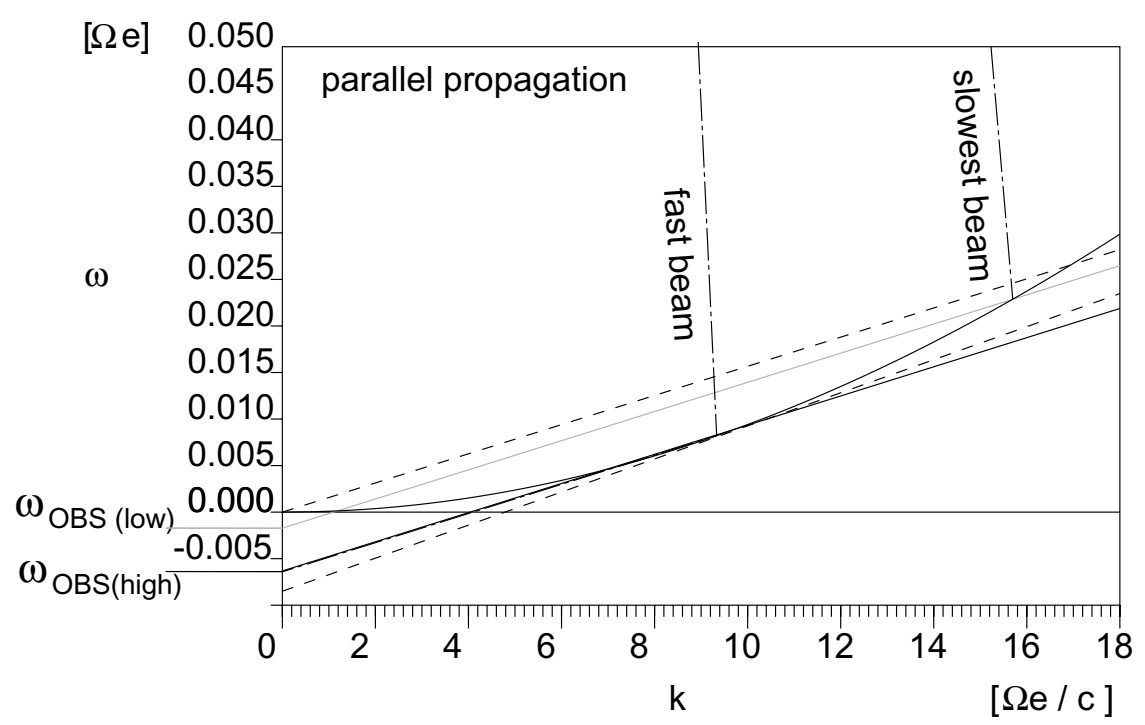

Fig. 11. The dispersion curve for the observed parameters $\frac{\omega_{p e}}{\Omega_{e}}=103, \theta=0, V_{S W}=502\left(\mathrm{~km} \mathrm{~s}^{-1}\right)$ and $\cos \theta_{k S}=0.94$, together with the lines representing Eq. (1). The light line indicates the wave detected at lowest frequency $\left|\omega_{O B S}\right|$, and the thick line indicates the wave detected at highest frequency $\left|\omega_{O B S}\right|$. The dashed lines with inclinations $V_{S W} \cos \theta_{k S}$ (upper) and $V_{S W} / \cos \theta_{k S}$ (lower) intersect the dispersion curve at the upper and lower limits of frequency range, respectively.

Oct. 25, 1994 GEOTAIL MGF LEP
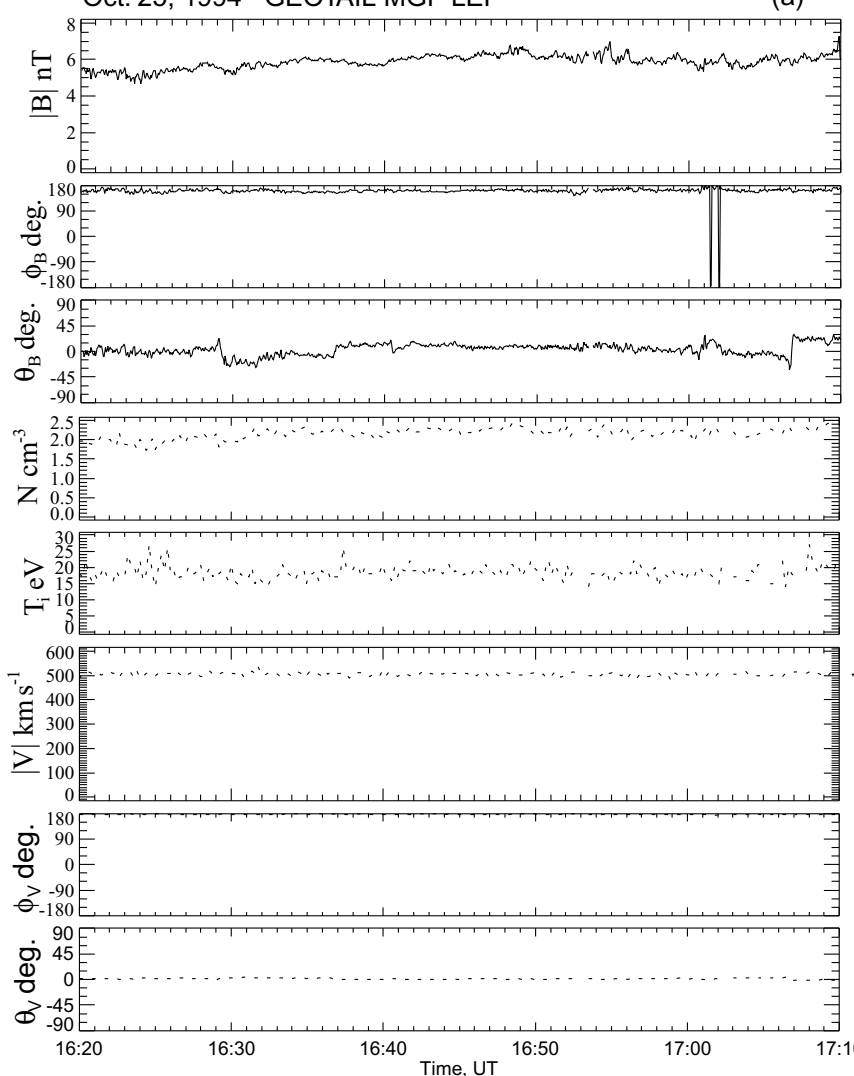

(a)
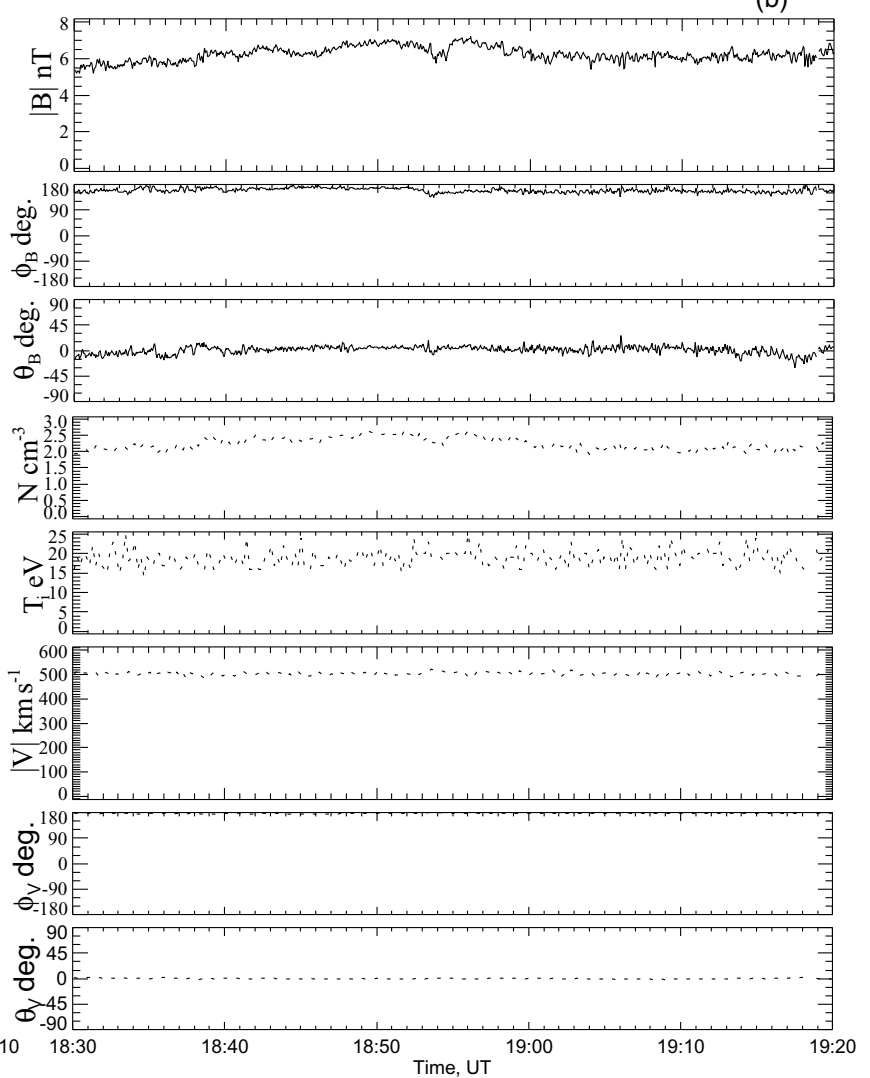

Fig. 12. The solar wind condition on October 25, 1994, for the periods of detection of the wake-related ULF waves. From top to bottom: the magnitude $|B|$ and solar ecliptic angles $\phi_{\mathrm{B}}, \theta_{\mathrm{B}}$ of the interplanetary magnetic field, ion number density $N$, ion temperatude $T_{\mathrm{i}}$, the magnitude $|V|$ and direction $\phi_{\mathrm{V}}$, $\theta_{\mathrm{V}}$ of the solar wind bulk velocity. The original figures were created by the DARTS system at ISAS, Japan.

the lowest energy of the resonant electrons is calculated to be $0.062 c$, which corresponds to $0.96(\mathrm{keV})$.

At the beginning and the end of each event, the lower boundary of $\left|\omega_{O B S}\right|$ was as high as the upper boundary. It indicates that the the lowest energy of the resonant electrons was as high as $2.5(\mathrm{keV})$. When the lowest energy of the resonant electrons became higher than that, the resonant wave became inaccessible to GEOTAIL as the group velocity was too small to propagate upstream against the solar wind flow. This is the way how the ULF fluctuations disappeared from 


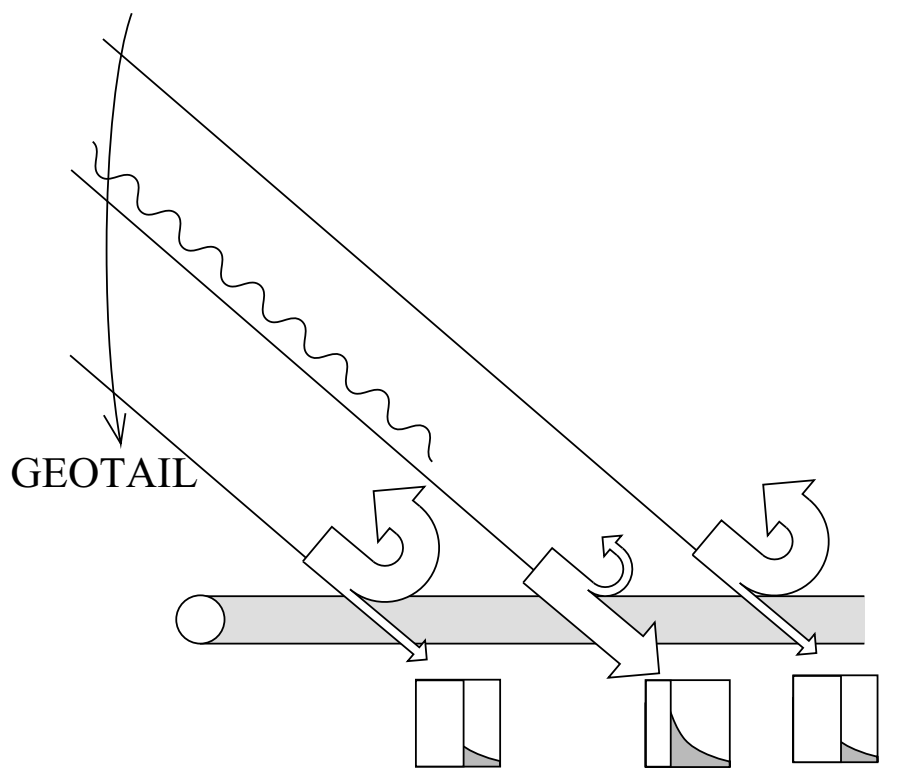

Fig. 13. A schematic illustration of spatial variation of potential structure of the boundary of the lunar wake (not in scale). In most parts of the wake boundary, the potential difference is large enough to reflect low-energy electrons, but in some areas, the potential drop is reduced, and some components of lower energy electrons can pass through the lunar wake and undergo resonant interaction with whistler mode waves with a group velocity large enough to propagate upstream against the solar wind. GEOTAIL detects the resonant wave intermittently, depending on which part of the wake the spacecraft was magnetically connected.

Table 3. Cycrotron resonance with ions.

\begin{tabular}{|c|c|c|c|}
\hline \multicolumn{4}{|c|}{ Wave in solar wind frame } \\
\hline Polarization & $\mathbf{k}$ & Ion beam & Resonance condition \\
\hline \multirow[t]{2}{*}{$\begin{array}{l}\text { left-hand } \\
\text { (ions) }\end{array}$} & $\begin{array}{l}\text { anti- } \\
\text { sunward }\end{array}$ & sunward & $\begin{array}{l}\omega+\left|\mathbf{k} \mathbf{V}_{B E A M}\right|+\left|\mathbf{k} \mathbf{V}_{S W}\right|=n \Omega_{i} \\
\mathrm{n}>10, \text { improbable }\end{array}$ \\
\hline & & $\begin{array}{l}\text { anti- } \\
\text { sunward }\end{array}$ & $\begin{array}{l}\omega-\left|\mathbf{k} \mathbf{V}_{B E A M}\right|+\left|\mathbf{k} \mathbf{V}_{S W}\right|=n \Omega_{i} \\
\text { not accessible from GEOTAIL }\end{array}$ \\
\hline
\end{tabular}

$\Omega_{i}$ : ion cycrotron frequency

the GEOTAIL observation during the period of stable solar wind.

The lowest energy of the resonant electrons is thought to be equal to the potential drop of the lunar wake. That is, the potential drop was smallest in the middle of each event, and higher at the beginning and the end of the events.

\section{Discussion}

\subsection{Variation in potential drop of the wake}

The variation in the lower boundary of $\left|\omega_{O B S}\right|$ is not due to variations in the solar wind parameters. A variation in solar wind speed $V_{S W}$ would cause variations in both upper and lower boundaries of the wave activity as reported by Farrell et al. (1996). For the cases presently analyzed, the solar wind was actually stable (Fig. 12) and only the lower boundary of $\left|\omega_{O B S}\right|$ varied with upper boundary unchanged.

Figure 10 shows that the lower limit of the observed frequency $\left|\omega_{O B S}\right|$ depends on the lowest energy of the resonant electrons, and thus the potential drop of the lunar wake. The fact that the lower limit of the observed frequency $\left|\omega_{O B S}\right|$ was lowest in the middle of each event indicates that the potential drop of the lunar wake was lowest in the middle of each event. At the beginning and the end of each event, the lower limit of $\left|\omega_{O B S}\right|$ was higher, and the potential drop was larger. It indicates that there was a spatial variation in potential structure of the lunar wake. Figure 13 illustrates the idea. On most of the wake boundary, the potential difference was large enough to reflect low-energy electrons. Only high energy components of electrons can pass through the wake and coupled with whistler mode waves at relatively smaller frequency around which the group velocity is not very fast, thus the resonant waves cannot propagate against the solar wind stream. In such areas of the wake boundary, there must be some area where the potential difference was small enough for lower energy electrons to pass through to undergo resonant interaction with whistler mode waves whose group velocity was large enough to propagate upstream of the solar wind. The orbital motion of GEOTAIL caused a change in geometry of magnetic connection to the wake; the resonant wave was observed intermittently when the spacecraft was connected to the region of reduced potential barrier.

\subsection{Testing possibility of ion cyclotron resonance}

In Section 3.2 we assumed that the ULF waves were righthanded whistler mode in the solar wind frame. Here we reexamine one of the other candidates. As the ULF waves were observed upstream of the Earth's bow shock, there is a possibility that ions reflected at the bow shock traveled far upstream from GEOTAIL, where they might excite waves. Ta- 
ble 3 summarizes the resonance condition for ion beams that can account for the left-hand polarization in the GEOTAIL frame of reference. In this case, the wave must propagate downstream in the solar wind, $\mathbf{k} \dot{\mathbf{V}}_{S W}>0$ as summarized in Table 1. For sunward streaming ions with a velocity $\mathbf{V}_{B E A M}$ in GEOTAIL frame of reference, the resonance condition with anti-sunward propagating, left-hand polarized wave in the solar wind frame of reference is

$$
\omega+\left|\mathbf{k} \mathbf{V}_{B E A M}\right|+\left|\mathbf{k} \mathbf{V}_{S W}\right|=n \Omega_{i},
$$

where $\Omega_{i}$ is the ion cyclotron frequency. By substituting Eq. (1), Equation (13) becomes

$$
\omega_{O B S}+\left|\mathbf{k} \mathbf{V}_{B E A M}\right|=n \Omega_{i}
$$

The observed frequency was $1.1 \mathrm{~Hz}$ while the ion cyclotron frequency was $0.09 \mathrm{~Hz}$, the number $n$ in Eqs. (13) and (14) becomes more than 12 . It is not likely that energy was effectively exchanged between waves and particles through a resonant interaction with such a large $n$.

On the other hand, if we think of ion beams flowing downstream, the resonant condition

$$
\omega-\left|\mathbf{k} \mathbf{V}_{B E A M}\right|+\left|\mathbf{k} \mathbf{V}_{S W}\right|=n \Omega_{i}
$$

can be satisfied with small $n$ by significant velocity $\mathbf{V}_{B E A M}$ of ions, but in such case, the resonant wave would be excited and propagate downstream of the bow shock, and is completely inaccessible to GEOTAIL.

Thus, the left-hand polarized ULF waves as detected by GEOTAIL cannot be explained by cyclotron resonance of left-handed wave with ions reflected by the bow shock.

\section{Summary and Conclusions}

ULF waves were detected by GEOTAIL at 27 lunar radii upstream of the moon, when the spacecraft was magnetically connected with the lunar wake. The frequency ranged from 0.3 to $1.1(\mathrm{~Hz})$ with sharp upper boundary. The ULF wave was propagating in a direction nearly parallel to the background magnetic field, and was lefthand polarized as measured in the spacecraft frame of reference. The wave is explained by reversal of polarization of right-handed, sunward-propagating electron whistler waves, excited through electron-cyclotron resonance with downstream-flowing electron beams. The downstream flowing electrons result from a filtering effect of the lunar wake. Low-energy components of electrons are reflected back by the potential drop at the lunar wake, and the rest components, with energies higher than that of the electric potential at the boundary of the lunar wake, penetrate through the wake into the downstream solar wind. The velocity distribution of downstream electrons would be modified to have some bump or shoulder to form a beam, which is likely to excite whistler mode wave through cyclotron resonance. The upper limit of the observed frequency was determined from the condition that the wave can propagate upstream against the solar wind. The lower boundary of the observed frequency indicates the lowest energy of the resonant electrons which ranged from $0.96(\mathrm{keV})$ to $2.5(\mathrm{keV})$. The lowest energy of the resonant electrons is a measure of potential barrier at the boundary of the lunar wake. The variation in the lowest frequency suggests that there are some regions where potential drop is reduced.

Acknowledgments. The authors are grateful to S. Kokubun and GEOTAIL/MGF team, who carried out the high-time resolution magnetic field measurement on GEOTAIL, and to T. Nagai, who supplied the 16- $\mathrm{Hz}$ sampling MGF data. Thanks are also due to H. Matsumoto, H. Kojima and GEOTAIL/PWI team for providing the plasma frequency on October 25, 1994. The authors thank S. Machida and $\mathrm{K}$. Tsuruda for valuable comments and discussions.

\section{References}

Bosqued, J. M., N. Lormant, H. Rème, C. d'Uston, R. P. Lin, K. A. Anderson, C. W. Carlson, R. E. Ergun, D. Larson, J. McFadden, M. P. McCarthy, G. K. Parks, T. R. Sanderson, and K.-P. Wenzel, Moon-solar wind interaction: First results from the WIND/3DP experiment, Geophys. Res. Lett., 23, 1259-1262, 1996.

Fairfield, D. H., Whistler waves observed upstream from collisionless shocks, J. Geophys. Res., 79, 1368-1378, 1974.

Farrell, W. M., R. J. Fitzenreiter, C. J. Owen, J. B. Byrnes, R. P. Lepping, K. W. Ogilvie, and F. Neubauer, Upstream ULF waves and energetic electrons associated with the lunar wake: Detection of precursor activity, Geophys. Res. Lett., 23, 1271-1274, 1996.

Futaana, Y., S. Machida, Y. Saito, A. Matsuoka, and H. Hayakawa, Counterstreaming electrons in the near vicinity of the moon observed by plasma instruments on board NOZOMI, J. Geophys. Res., 106, 18729-18740, 2001.

Kokubun, S., T. Yamamoto, M. H. Acunã, K. Hayashi, K. Shiokawa, and H. Kawano, The GEOTAIL magnetic field experiment, J. Geomag. Geoelectr. 46, 7-21, 1994.

Lepping, R. P. and K. W. Behannon, Magnetic field directional discontinuities, 1, Minimum variance errors, J. Geophys. Res., 85, 4695-4703, 1980 .

Matsumoto, H., I. Nagano, R. R. Anderson, H. Kojima, K. Hashimoto, M. Tsutsui, T. Okada, I. Kimura, Y. Omura, and M. Okada, Plasma wave observations with the GEOTAIL spacecraft, J. Geomag. Geoelectr., 46, 59-95, 1994.

Ness, N. F. and K. H. Schatten, Detection of interplanetary magnetic field fluctuations stimulated by the lunar wake, J. Geophys. Res., 74, 64256438, 1969

Ness, N. F., K. W. Behannon, H. E. Taylor, and Y. C. Whang, Perturbations of the interplanetary magnetic field by the lunar wake, J. Geophys. Res., 73, 3421-3440, 1968.

Ogilvie, K. W., J. T. Steinberg, R. T. Fitzenreiter, C. J. Owen, A. J. Lazarus, W. M. Farrell, and R. B. Torbert, Observations of the lunar plasma wake from the WIND spacecraft on December 27, 1994, Geophys. Res. Lett., 23, 1255-1258, 1996.

Owen, C. J., R. P. Lepping, K. W. Ogilvie, J. A. Slavin, W. M. Farrell, and J. B. Byrnes, The lunar wake at $6.8 \mathrm{R}_{L}$ : WIND magnetic field observations, Geophys. Res. Lett., 23, 1263-1266, 1996.

Schubert, G. and B. R. Lichtenstein, Observations of moon-plasma interactions by orbital and surface experiments, Rev. Geophys. Space Phys., 12, 592-626, 1974.

Sonnerup, B. U. Ö. and L. J. Cahill, Jr., Magnetopause structure and attitude from Explorer 12 observations, J. Geophys. Res., 72, 171-183, 1967.

T. Nakagawa (e-mail: nakagawa@tohtech.ac.jp), Y. Takahashi, and M. Iizima (e-mail: iizima@stpp1.geophys.tohoku.ac.jp) 